\title{
Meta
}

Journal des traducteurs

Translators' Journal

\section{(Im)possible coïncidence des textes : l'ordinaire de la création. " The Sea in Winter " (Derek Mahon) / " La Mer hivernale " (Jacques Chuto)}

\section{Maryvonne Boisseau}

Volume 62, numéro 3, décembre 2017

La traduction littéraire comme création

URI : https://id.erudit.org/iderudit/1043948ar

DOI : https://doi.org/10.7202/1043948ar

Aller au sommaire du numéro

Éditeur(s)

Les Presses de l’Université de Montréal

ISSN

0026-0452 (imprimé)

1492-1421 (numérique)

Découvrir la revue

Citer cet article

Boisseau, M. (2017). (Im)possible coïncidence des textes : l'ordinaire de la création. " The Sea in Winter " (Derek Mahon) / " La Mer hivernale " (Jacques Chuto). Meta, 62(3), 552-564. https://doi.org/10.7202/1043948ar
Résumé de l'article

La traduction littéraire n'est pas simple communication ou simple transmission du sens. Elle est aussi écriture originale parce qu'elle procède d'une énonciation qui est unique comme celle dont a procédé le texte initial. C'est en se fondant sur cette hypothèse que cet article interroge l'adéquation entre les termes de traduction et création et la portée de la comparaison "latraductioncomme création ". L'article s'attache d'abord à la proximité sémantique des termes de création et créativité que l'on substitue souvent l'un à l'autre. Puis sont examinés les liens entre le processus de traduction en tant que cheminement créatif et le " produit ", en tant que création. L’article avance l'idée que la traduction peut être appréciée comme une création si elle résulte d'un travail qui vise à la rencontre des textes et cherche une concordance esthétique et poétique entre les langues en puisant dans les profondeurs de la langue de traduction. À cette fin, sont comparés des extraits du poème « The Sea in Winter » de Derek Mahon et de sa traduction par Jacques Chuto, « La Mer hivernale ", afin de mettre au jour les ressorts de l'ordinaire de la création. 


\title{
(Im)possible coïncidence des textes: l'ordinaire de la création. «The Sea in Winter» (Derek Mahon) / «La Mer hivernale» (Jacques Chuto)
}

\author{
MARYVONNE BOISSEAU \\ Université de Strasbourg, Strasbourg, France* \\ maryvonne.boisseau@unistra.fr
}

\begin{abstract}
RÉSUMÉ
La traduction littéraire n'est pas simple communication ou simple transmission du sens. Elle est aussi écriture originale parce qu'elle procède d'une énonciation qui est unique comme celle dont a procédé le texte initial. C'est en se fondant sur cette hypothèse que cet article interroge l'adéquation entre les termes de traduction et création et la portée de la comparaison «la traduction comme création». L'article s'attache d'abord à la proximité sémantique des termes de création et créativité que l'on substitue souvent l'un à l'autre. Puis sont examinés les liens entre le processus de traduction en tant que cheminement créatif et le «produit», en tant que création. L'article avance l'idée que la traduction peut être appréciée comme une création si elle résulte d'un travail qui vise à la rencontre des textes et cherche une concordance esthétique et poétique entre les langues en puisant dans les profondeurs de la langue de traduction. À cette fin, sont comparés des extraits du poème «The Sea in Winter» de Derek Mahon et de sa traduction par Jacques Chuto, «La Mer hivernale», afin de mettre au jour les ressorts de l'ordinaire de la création.
\end{abstract}

\begin{abstract}
Literary translation is not merely communication or transmission of meaning. Insofar as it proceeds from a unique enunciative process, in the same way as the original text itself, it is akin to original writing. Starting from this hypothesis, the present paper considers the adequacy between the two terms creation and translation and the extent to which the comparison, translation as creation, applies. First the semantic closeness of the terms creation and creativity that are often used interchangeably in the field of Translation Studies are examined. Then, the connections between the translation process as creative development and its "result" perceived as creation are discussed. It is argued that it may be possible to read a translation as a creation if it results from an effort aiming at reducing the difference between the texts and seeking an aesthetic and poetic concordance between languages by drawing on the resources of the target language. To this end, excerpts from the poem "The Sea in Winter" by Derek Mahon, and its translation into French, "La mer hivernale," by Jacques Chuto are contrasted to bring to light the forces behind the ordinary task of creative translating.
\end{abstract}

\section{RESUMEN}

La traducción literaria no es simplemente un acto de comunicación o la mera transmisión de un sentido. También es un proceso de escritura original, ya que procede de una enunciación que es tan única como la que dio origen al texto inicial. Sobre la base de esta hipótesis, el presente artículo plantea la adecuación entre los términos traducción y creación, y el alcance de la comparación la traducción como creación. El artículo se centra primero en la proximidad semántica entre los términos creación y creatividad, que suelen confundirse. A continuación, se analiza la relación entre el proceso de traducción como itinerario creativo y el «producto» como creación. El artículo defiende la idea de que se 
puede apreciar la traducción como creación si resulta de una labor que busca el acercamiento entre los textos y una concordancia estética y poética entre las lenguas, aprovechando para ello los recursos profundos de la lengua meta. A fin de mostrar los resortes corrientes de la creación, la autora se vale de una comparación de varios extractos del poema «The Sea in Winter» de Derek Mahon, con su traducción «La mer hivernale» por Jacques Chuto.

\section{MOTS CLÉS/KEYWORDS/PALABRAS CLAVE}

traduction littéraire, création, processus créatif, énonciation, concordance, rythme literary translation, creation, creative process, enunciation, concordance, rhythm traducción literaria, creación, proceso creativo, enunciación, concordancia, ritmo

\section{pour Jacques Chuto}

Il faut évidemment traduire dans la langue même que l'on parle, quitte à visiter celle-ci dans ses obscures mais belles profondeurs, lesquelles, pour n'être pas reconnues par les emplois quotidiens, n'en sont pas moins prêtes à répondre à l'appel même de la poésie.

(Yves Bonnefoy 2007: 8)

N'y aurait-il pas quelque démesure, quelque vanité, quelque folie à vouloir effacer le lien qui attache toute traduction au texte dont elle dérive? à vouloir faire d'une traduction une création? à prétendre, à soutenir même que la traduction est création? Ce sont bien les questions que l'on se pose devant l'invitation à considérer «la traduction littéraire comme création " et c'est la complexité redoutable de cette simple comparaison que cet article tentera, non pas de simplifier, mais de comprendre afin de mettre en lumière comment un lecteur peut rejoindre le traducteur dans son effort de transmettre une double expérience, celle d'une lecture et celle du traduire. Il s'agira d'abord d'interroger la relation entre les termes de traduction et création, puis d'envisager la création comme mise en œuvre d'un processus d'élaboration du sens et de mise en forme linguistique, dépendant des ressorts de la créativité dans une situation ordinaire de traduction. À cette fin seront comparés des extraits du long poème épistolaire de Derek Mahon intitulé «The Sea in Winter» (2011) et sa traduction par Jacques Chuto, «La Mer hivernale» (2013) ${ }^{1}$.

\section{1. «La traduction littéraire comme création»}

En premier lieu, on soulignera que c'est bien, dans ce titre, la traduction littéraire, en tant que genre de traduction par opposition à la traduction technique et spécialisée, plus communément maintenant appelée pragmatique (Froliger 2013), qui s’impose dans sa relation avec le terme création. Et si d'aucuns défendent l'idée que le travail de traduction d'un texte pragmatique n'est pas complètement éloigné d'un travail de création, les différences essentielles de fonction, de genre, de registre, de destinataires entre ces deux grandes familles de textes induisent alors des approches créatives sans doute bien éloignées les unes des autres dans leurs ressorts, sinon les processus qu'elles engagent. En second lieu, l'ordre linéaire inévitable de la proposition la traduction littéraire comme création et notre sens de lecture habituel, de gauche à droite, organisent la saisie de l'ensemble à partir du mot traduction vers le mot création, nous amenant à penser intuitivement la traduction - au sens ici de transport d'un texte 
écrit en une langue donnée vers une autre langue - dans l'immédiat de cette appréhension globale, comme un processus de création, autrement dit d'invention, de surgissement - un mouvement vers du nouveau, de l'inattendu. Ce qui n'est qu'une première impression de lecture pourrait se formuler autrement comme lorsqu'on déclare: il y a de la créativité dans l'activité de traduction, voulant dire il y a forcément de la créativité dans l'activité de traduction; et, disant cela, on laisse aussi entendre qu'il n'y a pas que cela, que la créativité fait partie d'un ensemble de choses qui se conjuguent pour constituer un processus cognitif complexe, plus ou moins régulé selon le type de textes que l'on traduit. Et puis, voilà que subrepticement, l'on est passé de création à créativité. Aussi, lorsque l'on pense création en relation avec la traduction, on envisage le plus souvent la créativité, au sens de pouvoir créatif, de capacité mesurable et susceptible d'être évaluée plutôt que le produit lui-même, plutôt que la traduction. C'est comme si, avant de reconnaitre la traduction comme création, il fallait nécessairement en passer par un repérage de ces éléments (critères? paramètres? traces textuelles?) qui signalent une approche créative et talentueuse de la traduction par opposition à une approche banale, ordinaire, laborieuse. La créativité apparaît alors comme étant inhérente au processus de recherche de la solution esthétiquement optimale à un problème ou, plus exactement, à une difficulté de traduction ${ }^{2}$, dans quelque domaine que ce soit, mais cette capacité créative est plus ou moins visible, plus ou moins partagée. C’est ainsi que Folkart (1991 : 417) distingue la «traduction mimétique» de ce qu'elle appelle «la création traductionnelle». Il y aurait donc des degrés dans la créativité et, de ce fait, la traduction serait plus ou moins création. Elle le serait nécessairement, cela va presque sans dire, dans le cas de la poésie: «C'est de toute évidence à propos du texte poétique que la problématique de la traduction comme création se pose avec le plus d'acuité» (Folkart 1991: 417). On retrouve dans cette formulation de Folkart la comparaison des deux activités qui, l'une et l'autre, impliquent un faire et, par conséquent un savoir-faire ainsi que la maîtrise des outils et instruments indispensables au traducteur, en l'occurrence la langue de traduction dans tous ses arcanes. Le savoir-faire en question est donc un savoir-faire langagier impliquant cognition, connaissances, expérience et sensibilité, autrement dit du cognitif, du cérébral et de l'affect.

De plus, l'adverbe comme, qui sépare tout autant qu'il relie les deux termes, invite à défaire la lecture linéaire parce qu'il place sur un même plan et à égalité ces deux activités en les comparant tout en les identifiant l'une à l'autre sans pour autant les confondre, si bien que le mouvement est réversible: le second terme (création) devient le repère du premier (traduction). Cette différence formelle de statut entre les deux termes (comparé/comparant) confirme l'inversion à peine consciente du sens de lecture: la création comme traduction. Le caractère réversible de ce rapport induit en effet un rapprochement paradoxal des deux termes qui se trouvent alors dans un rapport ambivalent et contradictoire d'identification et de comparaison (en termes ici d'opérations et de valeurs énonciatives). Plus qu'une ressemblance, plus qu'une parenté, il y aurait donc, pour ainsi dire entre traduction - littéraire en l'occurrence - et création un double rapport de synonymie et de complémentarité. Toutefois, le besoin de souligner par un comme de comparaison et d'identification (identifying comparison, dirait-on en anglais) ce rapport que l'on voudrait fusionnel implique que ce dernier ne va pas de soi: les termes de traduction et création ne se substituent pas l'un à l'autre. Ce rapport n'est pas donné mais construit. Il faut en effet que se réalise 
une espèce d'assimilation de l'une à l'autre, qu'on ne peut guère imaginer, sauf à considérer qu'il existe une affinité profonde entre des processus qui, dans les deux cas, engagent un travail, de la matière - quand le langage poétique, dans son épaisseur, se fait matériau -, du temps et un espace où se déployer. Cet espace est la différence des langues, leur irréductibilité les unes aux autres, même lorsqu'elles sont proches comme peuvent l'être l'anglais et le français, matérialisée par un écart, autrement dit leur non-concordance. Condition nécessaire à l'exercice de la traduction, cet écart est le lieu même de la «création traductionnelle» (Folkart 1991: 417), magnifiée d'une part dans l'expérience d'une coïncidence - au sens de rencontre parfois possible, mais le plus souvent impossible, non plus des langues - qui ne sont pas superposables quand bien même elles sont proches -, mais des textes travaillés et, d'autre part dans la recherche d'une concordance esthétique et poétique d'une langue à l'autre, d'une forme à l'autre. La problématique n'est plus ici celle de l'équivalence en traduction mais plutôt celle d'un accord résultant de l'interprétation, et celle, étrangement peut-être, d'une analogie (le rapport entre les deux textes est, lorsqu'on les compare, de l'ordre d'une correspondance, d'une identité seulement partielle), étant entendu que l'un des deux textes est la cause de l'autre, à l'origine de sa production, de sa création.

\section{Du processus au produit}

Envisageons alors le processus : la traduction est, à cet égard, une énonciation dont la particularité est de reposer sur une énonciation antérieure qui, d'un point de vue linguistique, en constitue la situation et le contexte, auxquels se superposent la situation et le contexte pragmatiques dans lesquels se trouve le traducteur. La situation d'énonciation nouvelle se déploie à partir d'un texte établi, de sa lecture et de son interprétation dans un contexte "ergonomique», le contexte de travail du traducteur (Lavault-Olléon 2011) qui n'est pas sans incidence sur sa plus ou moins grande capacité à mobiliser ce qu'on pourrait appeler la réserve créative du texte lui-même.

Par ailleurs, considérant qu'une énonciation est un événement linguistique unique - un même énoncé répété n'est déjà plus le même lors de sa répétition - on peut alors avancer l'idée que, du point de vue du traducteur-énonciateur, le texte produit, du seul fait de son caractère unique, est une création: on peut le reproduire, en faire une copie, mais son énonciation, au sens abstrait du terme de mise en mouvement de la langue impliquant un ensemble régulé d'opérations énonciatives, reste unique. Tout énoncé produit dans une situation banale de locution n'est cependant pas nécessairement une création langagière au sens esthétique ou artistique du terme. Il n'en reste pas moins que les hésitations, les tentatives et les remords du traducteur qui s'efforce de s'approcher au plus près d'une adéquation maximale, ou «relevante » (Derrida 1999), font partie (are part and parcel) de ce geste unique qu'est l'énonciation et qu'ils s'inscrivent dans l'activité métalinguistique d'un sujet énonciateur.

Le texte énoncé (et l'on voit bien que si l'on peut mentalement distinguer entre le processus et le produit, on glisse constamment de l'un à l'autre quand on parle de traduction, le terme lui-même étant porteur de cette ambivalence, disant l'action et son résultat ensemble dans le même mot), le texte, donc, est aussi création à chaque lecture - alors qu'on pourrait être tenté de dire re-création - dans la mesure où la lecture est, elle aussi, un autre mode de l'énonciation: le lecteur énonce un texte 
chaque fois qu'il l'écoute ou le lit et, de la même façon qu'un énoncé isolé n'est déjà plus le même lorsqu'il est répété, ce texte (terme qui, dans la métalangue linguistique, renvoie à toute production orale ou écrite, à un énoncé) n'est plus tout à fait le même non plus lorsqu'il est relu alors que sa forme n'a pas varié, sinon peut-être dans ce qu'on pourrait appeler son intonation intérieure. Ce texte nouvellement énoncé se distingue ainsi du texte énonçable, c'est-à-dire de l'original, à la fois énoncé et énonçable quand on pense à la traduction qu'il a appelée.

Penser la lecture comme un autre mode de l'énonciation fait alors apercevoir la complexité du processus et la position délicate du traducteur puisqu'il est d'abord lecteur lisant pour traduire et qu'il va ainsi devoir ajuster à sa mesure deux modes de l'énonciation. Il est à ce titre doublement créateur, à la fois interprète (il crée le sens du texte en l'interprétant ou, en renversant la formule, le sens du texte se construit dans et par l'interprétation), et écrivain (c'est dans sa langue qu'il énonce le texte d'un autre, par la lecture, puis par l'écriture (on parle communément, dans ce cas, d'écriture seconde ou de ré-écriture), que cette écriture soit oralisée ou non (prononcée intérieurement en même temps qu'écrite). C'est alors dans sa langue que l'activité créatrice va laisser des traces mais, si l'on peut tenter de découvrir les signes de cette confrontation des langues et des textes, sans doute est-il vain de chercher à théoriser l'acte même de création qui ne peut sourdre que du rapport singulier que le traducteur entretient avec sa propre langue. Plus modestement, ce sont donc ces traces textuelles que l'on peut repérer et décrire pour s'efforcer de comprendre cette part créative du processus de traduction et montrer qu'elle s'inscrit dans le texte traduit comme une signature du traducteur:

Que peut supporter la langue qui est la mienne lorsque la langue étrangère et pourtant proche la scrute, la dilate, la met à mal, exige d'elle des raisons? Ce qui survient alors, le choix qui sera le mien, ce que je m'autorise et ce que je m'interdis, est le reflet très exact, j'oserai dire implacable, du rapport que j'entretiens avec ma langue. En termes plus précis, ce choix signe mon écriture et la situe, que je sois induit [...] à demeurer au plus près de l'original, ou [...] à m'écarter plus nettement d'une littéralité jugée non signifiante [...]. (Simeone 2014: 18)

Paroles de traducteur - c'est une écriture que le lecteur auquel est destinée la traduction reconnaîtra; c'est un travail de la langue et sur la langue que le lecteurtraductologue scrutera, à l'écoute du traducteur, mais aussi de l'auteur et en pleine connaissance de la langue étrangère, afin de comprendre comment la création se manifeste dans le nouveau poème. On notera là toute l'ambiguïté de l'entreprise puisque l'énonciation même n'est accessible que par l'intermédiaire de l'énoncé produit et ce que l'on va supposer être les traces mêmes d'un processus qui mêle tout à la fois les réflexes langagiers mus par la familiarité d'une langue partagée et l'expression singulière suscitée par ce que le traducteur «s'autorise» et "s'interdit», ce qui suppose un rapport intime à la langue que nous sommes tentée de qualifier d'amoureux. Dans le premier cas, on rapprochera lesdits réflexes de ce que linguistes et traductologues identifient comme des automatismes ou tendances du fait d'un fonds commun partagé par une communauté de locuteurs donnée (à laquelle appartient le lecteur, qu'il soit ce lecteur ordinaire, critique ou traductologue). Dans le second cas, on regardera sans doute cette singularité comme une marque stylistique individuelle, idiosyncratique.

Comment alors faire la part de ce qui relève du réflexe d'un côté, de ce qui procède de la réflexion de l'autre, l'un bousculant l'autre, quand l'accès au processus n'est 
qu'indirect? Dancette et al. (2007), dans le cadre d'une approche cognitive et à l'instar de modèles théoriques proposés dans d'autres domaines des sciences humaines et appliquées, suggèrent que «la créativité est reconnue par la valeur de la production et le processus qui l'engendre» (Dancette et al. 2007: 110) et distinguent des phases (phase préparatoire, incubation, proposition) qui sont autant de «moments de créativité au sein du processus de traduction» (Dancette et al. 2007: 111). Les auteurs en viennent à distinguer deux niveaux de créativité: celui de la "compréhension créative» et celui de la «réécriture créative» selon cinq axes: (intra)référentiel, formel, sémantique, narratif et traductologique, couvrant ainsi les contraintes du travail traductif, intégrant les plans du référentiel et de la forme. Cette démarche aboutit à l'établissement de "critères» qui s'appuient sur la façon dont les traducteurs non seulement s'approprient ces contraintes mais jugent du processus et du résultat. Bien que cela ne soit pas explicitement dit, ces critères - aboutissement, satisfaction, évaluation, résilience, reconnaissance des exigences élevées du domaine (Dancette et al. 2007: 119) - sont tous à double entrée, une entrée appréciation du traducteur et une entrée texte: au sentiment de travail abouti correspond la traduction aboutie; à la satisfaction éprouvée correspond le rendu acceptable de tel ou tel aspect du texte; à l'évaluation correspond l'amélioration des différentes versions du texte; à la résilience correspond le temps de résistance du texte; enfin, à la reconnaissance des exigences correspond la reconnaissance des difficultés ou de la valeur (littéraire) du texte. Cette recherche de critères permettant de déterminer si la traduction est création s'appuie donc sur la distinction traditionnelle entre forme et contenu, sur les dispositions psychologiques du traducteur lui-même et, implicitement, sur les aspects du texte susceptibles de susciter la créativité et donnant lieu à des solutions à la fois nouvelles et appropriées. En somme, elle s'attache davantage à la notion même de créativité, en tentant d'objectiver le travail conscient et raisonné du traducteur, qu'au paradigme d'une potentielle équivalence entre les deux termes, traduction et création. Or, pour apprécier cette relation étroite - et, nous l'avons dit, réversible - entre les deux, sans doute convient-il de garder à l'esprit que traduction et original sont liés par un «contrat fondamental» (Berman 1999: 40): «Ce contrat - certes draconien - interdit tout dépassement de la texture de l'original.»Et, comme le résume également Hewson (2006), le texte cible n'est finalement que le résultat d'un processus interrompu au moment où le traducteur a tranché pour telle ou telle solution ${ }^{3}$. La création n'est pas seulement innovation, trouvaille heureuse, figure de style ou réduction de la différence des langues: elle est dans l'écart assumé entre plusieurs choix motivés par le contexte et certaines contraintes discursives propres à la langue de traduction; elle est «dans la langue même que l'on parle» (Bonnefoy 2007: 8). C'est ce que nous appelons l'ordinaire de la création, tributaire d'un déjà-dit, d'un déjà-là. Et, s'il y a bien du jeu dans la relation entre le déjà-dit du texte à traduire et le traducteur, la réserve qu'impose ce texte contient le désir d'une créativité recherchée pour ellemême. Cette limite cependant renvoie à l'exigence d'un travail au plus près du texte original et de la langue qui en renverra une image. Le résultat de ce travail n'est pas forcément spectaculaire ni ne s'affiche comme nécessairement innovant. Il n'en révèle pas moins une maîtrise sans laquelle il n'y a pas de création, ce qu'illustrent les quelques échantillons extraits de "The Sea in Winter» et de sa traduction, «La mer hivernale», analysés ici. 


\section{Impossible coïncidence : "The Sea in Winter", "La mer hivernale»}

«The Sea in Winter» est un long poème épistolaire de douze strophes de huit vers chacune dans sa version définitive traduite par Chuto (la première version publiée en 1979 compte vingt-deux strophes). Il est dédié et adressé à un autre poète, Desmond O'Grady, qui, à l'époque où le poème a été écrit, vivait sur l'île de Paros mentionnée dans la troisième strophe du poème ("the dancing lights of Náousa ») et Mahon, alors en résidence à l'Université de Coleraine où il tente d'enseigner et qui vit dans un meublé à Portrush, transforme la petite station balnéaire où il habite et les bords de mer ventés de la côte nord de l'Irlande en rivages de lumière éclairant un avenir lointain qu'il voudrait idéal («ideal», strophe 10). Se trouvent réunis et superposés deux espaces géographiques que tout oppose: une réalité sombre, étrange ("weird», strophe 9) se désigne comme si elle était autre, et «ce qu'elle "n'est pas" (ou sa part de non-être) devient une source de propositions possibles pour la fiction et la poésie» (Dayre 2009: 236). Du point de vue du sens, la poésie naît d'une forme de spleen lié à la situation personnelle du poète et du contraste entre un univers déprimant et la réalité transformée par la lumière venue d'ailleurs. Du point de vue de la forme, elle naît de la succession de strophes régulières marquant le rythme monotone des jours et des nuits, celui des saisons et de la vie perdue "au bord de l'espace», source possible néanmoins d'un nouvel élan (strophe 9). L'original et sa traduction sont présentés en vis-à-vis, l'original sur la page de gauche, la traduction sur la page de droite, offrant ainsi la traduction en toute transparence et la possibilité d'un vaet-vient d'un texte à l'autre.

Les limites de cette étude ne permettent pas un examen détaillé de l'ensemble de ce long poème qui oscille entre un rythme ternaire (trois parties de quatre strophes correspondant à une argumentation en trois points) et un rythme à quatre temps (quatre parties de trois strophes correspondant à un va-et-vient énonciatif entre le poète-énonciateur et son destinataire au sein duquel s'intercalent deux moments de généralisation). Nous concentrerons nos observations sur la partie médiane d'une division en trois parties, en particulier parce qu'elle se termine par la strophe dont les premiers mots constituent le titre du poème. On y rencontre des exemples de ce qui apparaît comme des choix naturels, coulant de source (il nous semble que le qualificatif automatique ne s'applique pas à la poésie même si ces choix sont en conformité avec des tendances linguistiques avérées), ne manifestant sans doute pas, aux yeux du lecteur ordinaire, de façon éclatante une créativité à l'œuvre; on y trouve également des exemples de choix plus marqués que l'on peut interpréter comme des indices du processus créatif même s'il est difficile, voire impossible, de concevoir, dans le fil du processus, une séparation entre ce qui serait un moment créatif et un autre qui ne le serait pas, la décomposition de l'énonciation en opérations n'étant qu'une façon d'objectiver et d'expliquer un cheminement.

\subsection{Proximité}

(1) Portstewart, Portrush, Portballintrae un beau pays mal habité, policed by rednecks in dark cloth and roving gangs of Tartan youth. 
Portstewart, Portrush, Portballintrae

un beau pays mal habité,

patrouillé par des péquenauds en drap sombre

et des bandes de jeunes en tartan qui vadrouillent.

Ces quatre premiers vers de la cinquième strophe ne présentent pas de «difficulté» et, comme de nombreux autres passages du poème, sont traduits au plus près de l'original - à la longueur du vers près ici - illustrant la transposition fréquente du participe présent en proposition relative, l'un et l'autre syntagmes qualifiant le nom. On comprend qu'il eût été maladroit d'adopter en vadrouille en raison de la similitude structurelle de l'expression en tartan qui traduit l'emploi adjectival de tartan et dont le choix contraint permet d'établir un parallèle avec le syntagme nominal du vers précédent: péquenauds en drap sombre/bandes de jeunes en tartan. Ce parallélisme compense-t-il l'allongement du vers en français? Le traducteur a-t-il établi ce parallélisme délibérément ou l'usage et le rythme du français ont-ils naturellement guidé la traduction? Une lecture plus fine et une écoute du rythme des deux langues permettent de répondre à ces questions par l'affirmative. La répétition de la première syllabe accentuée des toponymes crée une réverbération du son, dans le premier vers, légèrement déformé ensuite, mais à peine affaibli par la reprise de la consonne plosive [p] à l'initiale de pays puis de policed et patrouillé, tous deux en début de vers. L'écho s'éloigne, se déforme et se prolonge, adouci en anglais, avec la rime approximative de cloth/youth tandis qu'en français, patrouillé et vadrouillent se répondent, l'un en début de vers, l'autre à la fin du vers suivant. La traduction-création se manifeste ici dans le jeu sonore et la compensation du glissement des plosives vers les fricatives non voisées $[\theta]$ de la rime approximative par un autre effet d'écho, non intrusif cependant du fait de la distance entre les mots (patrouillé/vadrouillent), pour traduire cette rime impossible à transposer en français.

(2) and a strange poetry of decay charms the condemned hotels by day,

while in the dark hours the rattle

of a cat knocking over a milk-bottle

on a distant doorstep by moonlight

can set you thinking half the night.

et l'étrange poésie du déclin

enchante tout le jour ces hôtels condamnés,

tandis que, dans la nuit, le fracas

d'une bouteille de lait renversée par un chat

sur un perron lointain éclairé par la lune

peut donner à penser jusqu'à l'aube.

Il s'agit, dans ce deuxième exemple, des six premiers vers de la strophe suivante et on notera là encore la proximité des deux textes car les changements effectués, tous conformes à un usage classique du français, viennent ici souligner la musicalité nostalgique de l'original: imbrication du syntagme prépositionnel tout le jour, mise en relief de dans la nuit, étoffement du syntagme nominal: éclairé par la lune, modulation temporelle: jusqu'à l'aube. Tous ces changements (agencement syntaxique, transposition, modulation) sont parfaitement repérés par les traductologues et, individuellement, ne semblent pas relever de ce qu'on entend généralement par création. Pourtant, 
ils mettent en lumière l'opposition du jour et de la nuit, et annoncent «l'épiphanie évasive de l'aube» de la strophe suivante. Mieux encore, la strophe tout entière se lit, en français, comme une longue phrase musicale aux notes liées par les enjambements comme dans un legato mélancolique pouvant évoquer les Vers Saturniens de Verlaine. Dans ces quelques vers, l'accent peu marqué du rythme syllabique du français plaide en faveur d'une lecture plus mélodique que celle des vers en anglais et, à cet égard, la traduction fait mieux entendre encore que l'original cette nostalgie que l'anglais exprime de manière moins sourde. $\mathrm{Si}$, lors d'une lecture rapide, les changements mentionnés ci-dessus semblent s'être imposés, motivés par le sens, le continu du langage et le contexte plus large des strophes précédentes et suivantes, la mélodie du français, en revanche, laisse dans la mémoire du lecteur une impression durable véritablement poétique.

(3) faith that the trivia doodled here will bear their fruit sometime, somewhere;

l'assurance que, quelque part, un jour,

les riens que l'on gribouille donneront des fruits,

(4) The something rotten in the state

infects the innocent;

Le quelque chose de pourri dans l'État

infecte les innocents;

Ces deux autres exemples confirment le souci d'une fidélité à la fois au texte original et au rythme de la langue française (calque de l'exemple 4, modulation grammaticale portant sur le groupe nominal complexe the trivia doodled here et déplacement des adverbes de lieu et de temps dans l'exemple 3). Cependant, comme dans les deux exemples précédents, cette conformité à l'usage n'a rien à voir avec la banalité d'une traduction sans relief car elle répond au classicisme de l'anglais de Mahon et révèle l'ajustement possible de la langue française aux contraintes de l'anglais.

\subsection{Inflexions, modulations}

(2) and a strange poetry of decay charms the condemned hotels by day, while in the dark hours the rattle of a cat knocking over a milk-bottle on a distant doorstep by moonlight can set you thinking half the night.

et l'étrange poésie du déclin enchante tout le jour ces hôtels condamnés, tandis que, dans la nuit, le fracas d'une bouteille de lait renversée par un chat sur un perron lointain éclairé par la lune peut donner à penser jusqu'à l'aube.

D'autres inflexions, qui semblent marquer une moindre proximité entre les deux textes, sont également observables dans l'exemple 2 reproduit ci-dessus. Le passage, 
en effet, de l'article indéfini a à l'article défini $l$ ', invite à relire les vers qui précèdent, et surtout, à écouter la langue: «[...] and yet / there is that Hebridean sunset // and a strange poetry of decay [...].» En anglais les deux strophes se lisent dans le continu de la phrase coordonnée; si le déterminant avait été the, d'une part la notion // strange poetry of decay //, faute d'être repérée dans le contexte précédent, paraissait incongrue et, d'autre part, la conjonction and et le déterminant the se heurtaient du fait de la succession des consonnes tandis que le déterminant a favorise le passage à l'accent tonique de l'adjectif strange. C'est l'inverse qui se produit en français: il y aurait eu un hiatus entre et et une et la solution retenue de l'article défini $l$ ', plus heureuse à l'oreille, facilite alors le passage du sombre (un beau pays mal habité) à un clair-obscur propice à la rêverie. Cependant, de ce fait, on a l'impression de basculer d'une situation spécifique repérée par rapport à la perception de l'énonciateur à une situation générique: on change de plan pour tendre vers la représentation d'une expérience partagée par tout un chacun. Ce basculement et ce changement de plan se produisent aussi en anglais, mais plus tard, puisque le pronom you du dernier vers (can set you thinking) est ici un indéfini à valeur générique alors que les occurrences précédentes du pronom référaient au destinataire du poème, O'Grady. Dans cette même strophe, le changement de diathèse, actif-passif, et donc l'inversion de point de vue aux deux mêmes vers, permet de conserver une rime (fracas/chat) même si le bruit (rattle/bottle) semble quelque peu atténué en français du fait de l'absence du son $[\mathrm{k}]$ dans chat. Il est clair que ces choix ne sont pas dus au hasard ni non plus à la seule intuition, mais procèdent d'une attention à chaque détail du texte original.

Les deux derniers exemples qui suivent n'offrent, semble-t-il, rien d'autre qu'une concordance aussi étroite que possible entre les deux textes et les deux langues. Le premier (exemple 5) complète les six vers de la sixième strophe citée dans l'exemple 2 et les deux vers de l'exemple 6 ouvrent la dernière strophe de la partie médiane observée (huitième strophe du poème). Ils présentent une analogie de structure groupe nominal sujet suivi du prédicat répartis également sur les deux vers, ce que reproduit fidèlement la traduction, et c'est sans doute cette concordance syntaxique qui frappe le lecteur en premier lieu:

(5) The moon of Nineveh and Tyre shines still on the Harbour Bar.

La lune de Ninive et de Tyr

Brille encore au-dessus du Café du Port.

(6) The sea in winter, where she walks, vents its displeasure on the rocks.

La mer hivernale, où celle-ci s'avance, Épanche son déplaisir sur les rochers.

[Le pronom she/celle-ci renvoie à the goddess/la déesse, mentionnée au vers précédent, le dernier de la strophe 7, l'anaphore assurant le continu d'une strophe à l'autre.]

On pourra cependant s'interroger sur la traduction, dans l'exemple 5, de still par encore. La position post-verbale de still le rend ambigu et on pourrait imaginer, qu'au lieu d'être un adverbe modifiant le verbe, il se rapporte à la lune, comme s'il s'agissait d'un adjectif prédicatif. Dans ce cas, l'imbrication d'un adjectif, entre virgules, serait 
envisageable: Éclaire, immobile, le Café du Port. Toutefois, cette interprétation rencontre deux obstacles: d'une part le choix de éclairé par la lune pour traduire by moonlight deux vers plus haut rend difficile une nouvelle occurrence de éclairé qui, de plus, affaiblirait le sens du verbe shine; d'autre part, il s'agit, fictivement, de cette lumière venue d'ailleurs éclairer la misère du poète et de ces rivages désolés ( Up here where the air is thinner, / in a draughty bungalow in Portstewart, // beside my 'distant northern sea' / I imagine a moon of Asia Minor [...]» // «Ici, dans l'air moins dense / d'un bungalow venteux de Portstewart, // au bord de ma 'lointaine mer septentrionale', / j'imagine une lune d'Asie Mineure [...]» [strophes 1 et 2 du poème]). Enfin, dans la traduction arrêtée, le choix de l'adverbe de durée, après le glissement à l'atemporalité et à la valeur générique des vers précédents («l'étrange poésie du déclin»), rétablit la perception de la situation spécifique du poème et du moment d'écriture repérés par rapport à l'énonciateur.

De même, si l'on cherche à comparer la traduction la mer hivernale (exemple 6) avec d'autres options, la mer en hiver (calque), la mer, l'hiver, là où celle-ci s'avance, ou bien l'hiver, la mer où celle-ci s'avance, on s'aperçoit vite que privilégier la saison, et donc, le temps et la période plutôt que la qualité de la mer (telle qu'elle est généralement en hiver, ce que nous dit hivernale) non seulement gauchirait le sens et l'interprétation mais détruirait un rythme construit sur le nombre identique de syllabes en anglais et en français dans cette première moitié du vers et les allitérations [w] / [s] dans la seconde. Aussi, la concordance, qui n'est donc pas seulement syntaxique, n'est-elle pas fortuite mais également rythmique, au sens meschonnicien du terme. Elle s'inscrit dans le continu du langage, prolonge l'original et se donne, pour ainsi dire, comme le seul choix possible. Sans doute est-ce là ce qu'il faut entendre par «la traduction comme création».

L’appréciation de la traduction repose sur des lecteurs divers, ordinaires, cultivés ou savants, «liseurs attentifs» (Larbaud 1997: 115). Ceux-là savent qu'il n’y a pas de traducteur infaillible ni non plus de critique infaillible. Ils savent aussi qu'une lecture est fragile. Toutefois, le traducteur littéraire, et tout spécialement le traducteur de poésie, est investi d'une responsabilité particulière, celle de distinguer la traductioncommunication de la traduction-création. Amener le lecteur à saisir la singularité de l'œuvre et la transmettre sans effacer les contraintes imposées par l'original et la langue de traduction exigent de s'être approprié tout autant ce qui résiste que ce qui semble couler de source afin d'en faire une énonciation nouvelle:

Traduire, c'est exercer à l'égard du texte original la plus grande rigueur possible, obéir à une illusion de maîtrise, pour, au bout du compte, écrire la traduction, c'est-à-dire s'en remettre à l'intensité d'une expérience qui suppose, au-delà des instruments et des codes, un acquiescement ou un abandon, qu'on le nomme inspiration, création ou simplement, faisant retour à l'étymologie de poiesis, «faire». Car «faire» une traduction n'est pas seulement utiliser des outils mais délimiter grâce à eux, dans le rapport qu'entretient le traducteur avec le texte, un domaine accessible à une méthode, domaine au-delà duquel commence la part d'écriture, qui se joue, en propre et totalement dans la langue d'accueil, et qui voit le traducteur ne rendre compte soudain, qu'à lui-même, c'est-à-dire, en l'occurrence, à son commerce avec la langue qui est la sienne. À ce moment de l'acte, le traducteur est bien pour l'essentiel, écrivain. Il ne peut en être autrement, sous peine d'ignorer le défi littéraire que pose toute traduction, et de se limiter à ne produire que des calques ou des simulacres. (Simeone 2014: 35) 
Le traducteur du recueil La Mer hivernale ne s'est pas contenté de traduire, il a aussi éclairé la lecture par une préface et des notes explicatives, choisissant ainsi d'amplifier l'expérience de la lecture de $s a$ traduction au moyen d'un appareil érudit sans que ce dernier soit envahissant. Ce faisant, il donne au lecteur qui ne connaîtrait pas l'anglais ou serait insuffisamment informé du contexte biographique, littéraire et culturel de la poésie de Mahon, un accès partiel au monde qui forme l'arrière-plan sur lequel se détache l'écriture. Libéré de ce qui pourrait demeurer obscur, le lecteur peut alors s'abandonner à la transparence de la traduction, ni «calque» ni «simulacre», mais traduction-création.

\section{REMERCIEMENTS}

Nous remercions de leurs observations les lecteurs qui ont évalué la première version de cette étude. Nous tenons également à remercier tout particulièrement Jacques Chuto d'avoir su lire «entre les lignes» du texte et de nous avoir permis de préciser des analyses qui seraient restées incomplètes sans ses remarques.

\section{NOTES}

* UR Linguistique, Langues, Parole, EA 1339 LiLPa, ER Fonctionnements discursifs et Traduction, Université de Strasbourg.

1. Mahon, Derek (2011/2013), La Mer hivernale et autres poèmes. (Traduit par Jacques Chuto) Le Chambon-sur-Lignon: Cheyne éditeur (p. 92-98 pour le poème et sa traduction) Cette édition bilingue s'accompagne de notes érudites visant à éclairer citations, allusions, emprunts, collages. Nous remercions Cheyne éditeur de nous avoir autorisée à reproduire les quelques extraits cités, et The Gallery Press pour la version originale, Mahon, Derek (2011): New Collected Poems. Oldcastle: Gallery Books, 106-108.

2. Pour la différence entre problème de traduction et difficulté, voir Boisseau (2016: 166-168).

3. "The temptation is to see the result as conclusive evidence of a process. Now, the target text, in this sense, is more an obstacle to understanding creativity than a clue as to its nature, as it is only evidence of a final result. It is only when one " opens out " the target text and examines the range of possible choices from which it was taken that one can have some insight into the creative process» (Hewson 2006: 61-62).

4. La bibliographie ne comprend que les ouvrages d'auteurs effectivement cités dans l'article ou auxquels nous sommes redevable d'une idée ou d'un emprunt.

\section{RÉFÉRENCES ${ }^{4}$}

Berman, Antoine (1999): La Traduction et la lettre ou l'auberge du lointain. Paris: Éditions du Seuil.

Boisseau, Maryvonne (2016): Lire et relire Jacqueline Guillemin-Flescher. In: Maryvonne Boisseau, Catherine Chauvin, Catherine Delesse et Yvon Keromnes, dir. Linguistique et Traductologie. Les enjeux d'une relation complexe. Arras: Artois Presses Université, 159-172.

Bonnefoy, Yves (2007): Le paradoxe du traducteur. Préface. In: Jacqueline Risset, dir. Traduction et mémoire poétique. Paris: Hermann Éditeurs, 7-15.

Dancette, Jeanne, Audet, Louise et Jay-Rayon, Laurence (2007): Axes et critères de la créativité en traduction. Meta. 52(1):108-122.

DAyre, Éric (2009): L'Absolu comparé. Littérature et traduction. Paris: Hermann Éditeurs.

DERridA, Jacques (1999): Qu'est-ce qu'une traduction «relevante»? In: Actes des Quinzièmes Assises de la traduction littéraire (Arles 1998). Arles: Atlas/Actes Sud, 21-48.

FolKART, Barbara (1991): Le Conflit des énonciations. Traduction et discours rapporté. L'univers des discours. Candiac: Les Éditions Balzac. 
Frellger, Nicolas (2013): Les noces de l'analogique et du numérique. Paris: Les Belles Lettres. Hewson, Lance (2006): The Vexed Question of Creativity in Translation. Palimpsestes. Horssérie:53-63.

Larbaud, Valery (1946/1997): Sous l'invocation de Saint-Jérôme. Paris: Gallimard.

LAVAUlT-OllÉON, Élizabeth (2011): L’ergonomie, nouveau paradigme pour la traductologie. ILCEA. 14:15p. Consulté le 19 avril 2016, http://ilcea.revues.org/1078.

Meschonnic, Henri (1999): Poétique du traduire. Lagrasse: Verdier.

Simeone, Bernard (2014): Écrire, traduire en métamorphose. Lagrasse: Verdier. 